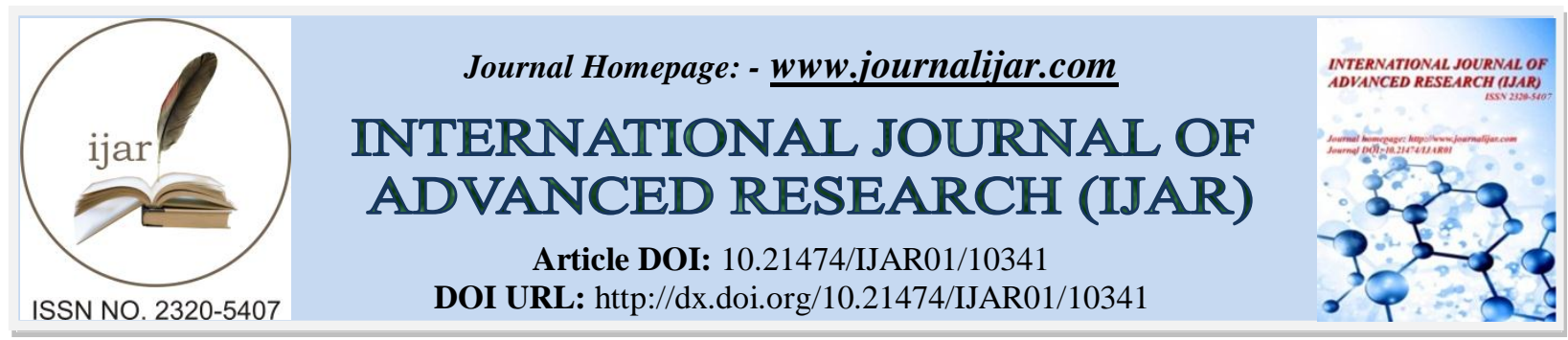

RESEARCH ARTICLE

\title{
HYPERPLASIE CONGENITALE DES SURRENALES (HCS) PAR DEFICIT EN 21-HYDROXYLASE : FORME VIRILISANTE PURE REVELEE TARDIVEMENT
}

\author{
M.El. Bahi, S. Rafi, G.El. Mghari and N.El. Ansari \\ Service De Diabétologie, Endocrinologie, Maladies Métaboliques Et Nutrition Hôpital Arrazi, CHU Mohammed \\ VI, Université Cadi Ayad, Marrakech.
}

\section{Manuscript Info}

………………….

Manuscript History

Received: 27 November 2019

Final Accepted: 30 December 2019

Published: January 2020

\section{Abstract}

Le deficit en 21 hydroxylase est la forme la pus frequente des hyperplasies congenitales des surrenales (hcs) ; elle constitue 95\% des hcs. Nous rapportons un cas rare d'une hyperplasie congenitale des surrenales par deficit complet en 21 hydroxylase dans sa forme virilisante pure revelee tardivement. C'est une patiente agee de 20 ans qui consulte pour une amenorrhee primaire, l'examen clinique a trouve un morphotype masculin avec un hirsutisme cote a 25 selon ferriman et gallwey, alopecie des golfes temporaux, une voie rauque, une hypertrophie du clitoris peniforme et orifice vaginale (prader iv), le tanner etait a s1 p5. Le dosage de la testosterone a 5,5ng/ml, du delta 4 androstendione a 42,8ng/ml (2,1-12.2) et de la17ohp a 193,6ng/ml, la cortisolemie etait basse a 2,3ug/dl. L'echographie pelvienne a trouve un uterus d'environ $3,78 \mathrm{~cm}$ avec les 2 ovaires de taille normale. La tdm abdominale a mis en evidence une hypertrophie homogene bilaterale des surrenales. La patiente a ete mise sous hydrocortisone $30 \mathrm{mg} / \mathrm{j}$ qui a permis le declenchement des cycles menstruels 6 mois apres le debut $\mathrm{du}$ traitement. A travers cette observation, nous insistons sur les caracteristiques cliniques et para cliniques de cette entite, ainsi que l'interet du diagnostic precoce pour traiter rapidement et prevenir d'eventuelles complications psychologiques tout en permettant une croissance normale, une puberte normale et une fertilite satisfaisante.

Copy Right, IJAR, 2020,. All rights reserved.

\section{Introduction:-}

L'hyperplasie congénitale des surrénales (HCS) est une maladie endocrinienne génétique à transmission autosomique récessive qui résulte du déficit d'une des enzymes de la stéroïdogenèse responsable de la synthèse des corticostéroïdes. Le déficit en 21 hydroxylase est la forme le pus fréquente elle constitue $95 \%$ des HCS. Nous rapportons un cas rare d'une hyperplasie congénitale des surrénales par déficit en 21 hydroxylase dans sa forme virilisante pure révélée tardivement.

\section{Cas clinique:-}

Patiente âgée de 20 ans qui consulte pour une aménorrhée primaire avec asthénie chronique sans amaigrissement ni notion d'épisodes d'hypotension ou d'hypoglycémie. L'examen clinique a trouvé un morphotype masculin avec des signes de virilisation (une pilosité pubienne de type masculine, des organes génitaux externes ambigus: des grandes lèvres présentes, une hypertrophie du clitoris et orifice vaginale (Prader IV), un hirsutisme coté à 25 selon Ferriman 
et Gallwey, alopécie des golfes temporaux, acné, séborrhée et une voie rauque. Le Tanner était à S1 P5. Le dosage de la testostérone à 5,5ng/ml, du delta 4 androstendione à 42,8ng/ml (2,1-12.2) et de la17OHP à 193,6ng/ml étant élevé a évoqué le diagnostic de l'hyperplasie congénitale des surrénales dans sa forme virilisante pure révélée tardivement.

La cortisolémie était basse à 2,3ug/dl. L’échographie pelvienne a trouvé un utérus d'environ $3,78 \mathrm{~cm}$ avec les 2 ovaires qui sont présents de taille normale. La TDM abdominale a mis en évidence une hypertrophie homogène bilatérale des surrénales. La patiente a été mise sous hydrocortisone $30 \mathrm{mg} / \mathrm{j}$ qui a permis le déclenchement des cycles menstruels 6 mois après le début du traitement.

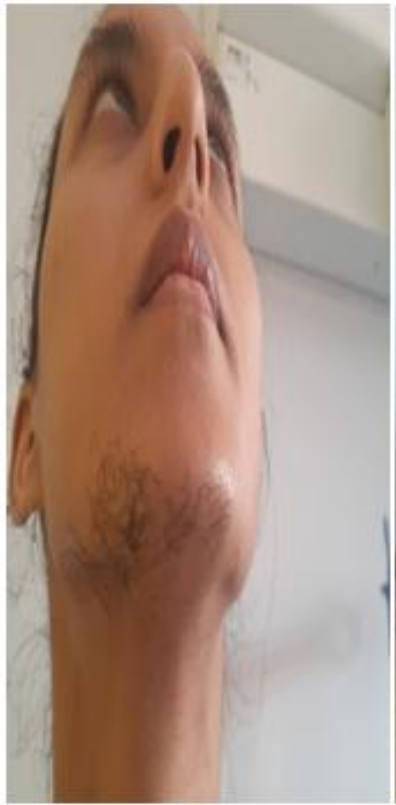

A

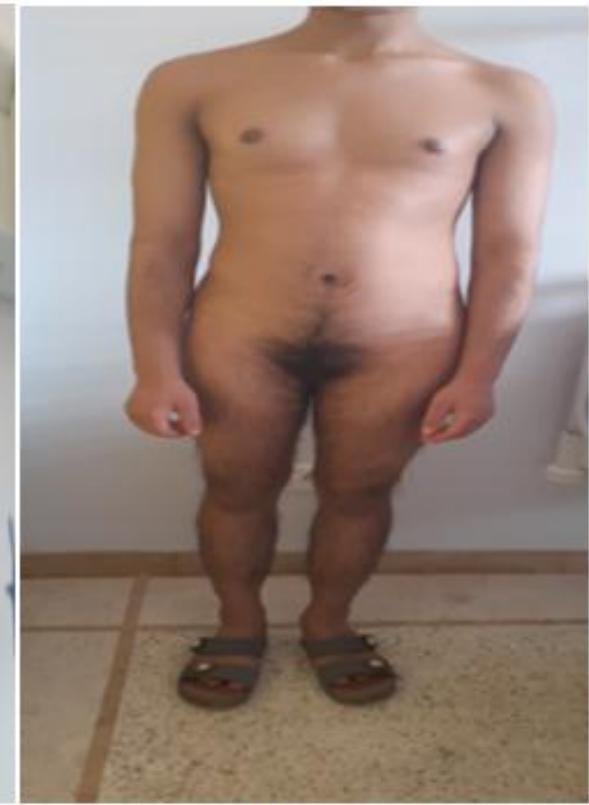

B

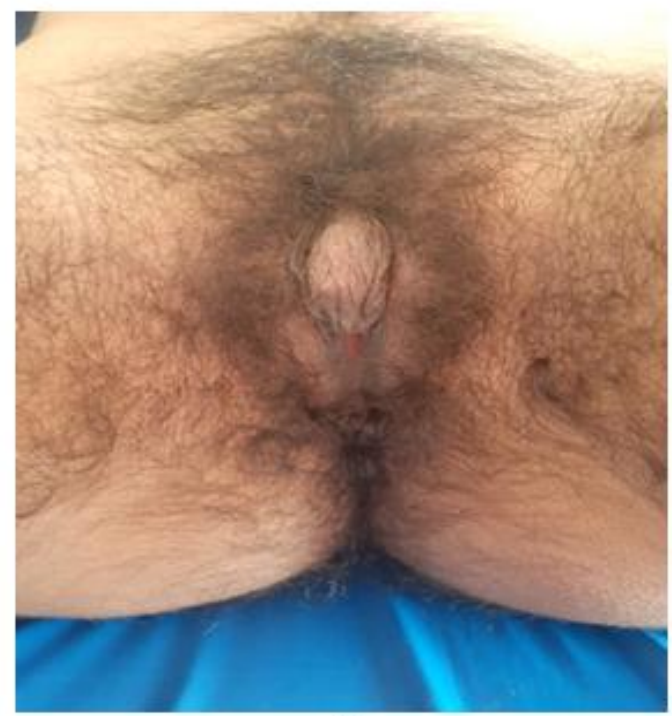

$\mathrm{C}$

Figure 1 :- Aspect d'hyper androgénie clinique dans le cadre d'une HCS dans sa forme virilisante pure découverte tardivement A : hursitisme sous mentonnier B : Morphotype masculin (hypertrophie musculaire, diamètre biacromial augmenté) $\mathrm{C}$ : Pilosité de type masculine et hypertrophie clitoridienne péniforme.

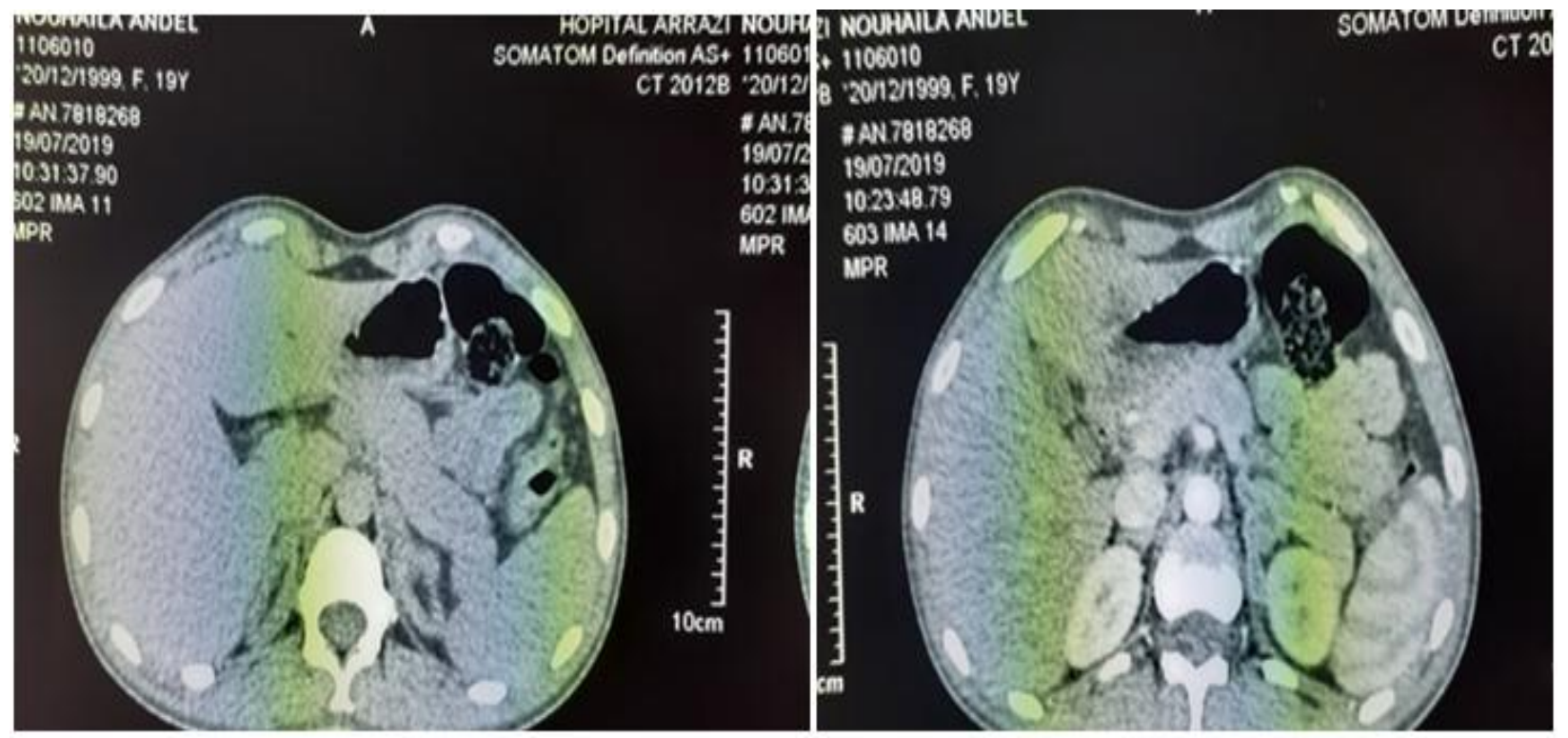

Figure 2:- Image IRM d'hyperplasie bilatérale des surrénales. 


\section{Discussion:-}

L'hyperplasie congénitale des surrénales est secondaire dans 90-95\% des cas au déficit de la 21 hydroxylase, en rapport avec des mutations du gène CYP21 A2 [1] à transmission autosomique récessive [2]. elle est caractérisée par un déficit en cortisol et un excès d'androgènes, accompagnés ou non d'un déficit en aldostérone.

Le déficit en 21-hydroxylase entraîne une insuffisance de la sécrétion de cortisol, une augmentation de la sécrétion d'ACTH responsable de l'hyperplasie surrénalienne, de l'accumulation du précurseur en amont du bloc enzymatique, la 17OHP, et d'une production excessive d'androgènes [2].

La sévérité de l'hyperplasie congénitale des surrénales dépend du degré du déficit en 21 hydroxylase. Les formes classiques se présentent souvent avec un syndrome de perte de sel (deux tiers des cas) dû à un déficit en aldostérone. Dans les autres cas, il s'agit de formes classiques virilisantes pures comme le cas de notre patiente[2]. Les filles porteuses de formes classiques d'HCS sont diagnostiquées en prénatal ou à la naissance, devant une virilisation des organes génitaux externes de degré variable (allant de l'hypertrophie clitoridienne à un aspect masculin des organes génitaux externes sans gonade palpable), avec un utérus et des ovaires normaux. Le diagnostic chez notre patiente était tardif jusqu'à l'âge de 20 ans devant l'aménorrhée primaire. L'aspect masculin et la clitoridomegalie étaient négligé par la patiente et son entourage notamment la mère.

La forme non classique est moins sévère, la présentation usuelle est celle d'une virilisation tardive parapubertaire ou postpubertaire [3].

Le diagnostic repose sur une élévation de la 17-hydroxyprogestérone sérique dont le taux de base permet toujours le diagnostic dans les déficits complets, come dans le cas de notre patiente $17 \mathrm{OHP}=193,6 \mathrm{ng} / \mathrm{ml}$. L'étude en biologie moléculaire du gène de la 21-hydroxylase (CYP21A2), gène situé sur le bras court du chromosome 6 , permet de préciser les différentes anomalies moléculaires possibles du gène [4].

L'hyperandrogénie étant un facteur perturbateur de l'axe gonadotrope, sera à l'origine d'une dysovulation ou une anovulation se traduisant par des troubles du cycle, une aménorrhée ou encore une infertilité [5].

L'échographie pelvienne confirme la présence de l'appareil génital féminin et trouve fréquemment un aspect échographique d'ovaires micropolykystiques secondaire à l'hyperandrogènie qui ne devrait pas prêter à confusion avec le syndrome des ovaires micropolykystiques qui est un diagnostic d'élimination [6].

Le traitement dépend des symptômes, en cas d'hirsutisme une dose modérée d'hydrocortisone (30 mg par jour) ou de déxaméthasone $(0,25$ à $0,5 \mathrm{mg}$ par jour) permet de freiner la sécrétion d'ACTH [7]. L'acétate de ciprotérone peut aussi être utilisé en association avec l'ethinyl-aestradiol et semble être plus efficace en absence de contre-indication. Les troubles du cycle sont traités par hydrocortisone et ou progestatif. Notre patiente a été mise sous hydrocortisone $30 \mathrm{mg} / \mathrm{j}$ qui a permis le déclenchement des cycles menstruels 6 mois après le début du traitement.

Le dépistage néonatal est essentiel dans le diagnostic de ses cas d'HCS, il est systématique en France et en USA réalisé au 3ème jour de vie (dosage de la 17-hydroxyprogestérone sur papier buvard) permet de prendre en charge précocement les enfants avec formes classiques d'HCS dans un service d'endocrinologie pédiatrique.

\section{Conclusion:-}

L'HCS à révélation tardive est l'un des diagnostic devant être systématiquement recherché devant des patientes adolescentes voir même adulte présentant des signes d'hyperandrogènie sévère avec virilisation associés ou non à des troubles du cycle ou à une infertilité. Le diagnostic doit être le plus précoce possible Permettant ainsi d'avoir une croissance normale, une puberté normale, une fertilité satisfaisante et un état psychologique stable.

\section{Bibliography:-}

1. Brigitte Letombe, Sophie Catteau-Jonard, Geoffroy Robin. Endocrinologie en gynécologie et obstétrique.Elsevier masson SAS Chapitre. 2012;12:139-148. [Google Scholar]

2. Galand-Portier MB, Kuttenn F. Hyperplasie congénitale des surrénales à révélation tardive par déficit partiel en 21 hydroxylase. EMC Elsevier Masson SAS Paris. Endocrinologie-Nutrition. 2007;25:10-015-B.[Google Scholar] 
3. Moran C, Knochenhauer ES, Azziz R. Non classic adrenal hyperplasia in hyperandrogenism: a reappraisal. J Endocrinal Invest. 1998;21:707-20. [PubMed] [Google Scholar]

4. Maguelone G. Forest, Recent advances in the diagnosis and management of congenital adrenal hyperplasia due to 21-hydroxylase deficiency, Human Reproduction Update, Volume 10, Issue 6, November/December 2004, Pages 469-485

5. Moran C, Azziz R, Weintrop N, Witchel SF, Rohmer V, Dewailly D, et al. Reproductive outcome of woman 21 hysroxylase-deficient non classic adrenal hyperplasia. J Clin Endocrinal Metab. 2006;91:3451-6. [PubMed

6. Brigitte Letombe, Sophie Catteau-Jonard, Geoffroy Robin. Endocrinologie en gynécologie et obstétrique.Elsevier masson SAS Chapitre. 2012;3:149-160. [Google Scholar]

7. Mahou Deau J. Trouble de la différenciation sexuelle. Ency Med Chir, Gynécologie. 1979;30:419-423. 